\title{
Potenzialabschätzung von Synergieeffekten zur simultanen Rückgewinnung von Wertmetallen aus komplexen, metallhaltigen Reststoffen
}

\author{
Eva Gerold · Helmut Antrekowitsch
}

Angenommen: 27. Oktober 2021 / Online publiziert: 25. November 2021

(c) Der/die Autor(en) 2021

Zusammenfassung Das Ziel dieser Arbeit ist die Abschätzung der Möglichkeiten einer simultanen Aufarbeitung von verschiedenen, metallhaltigen Reststoffströmen aus der Industrie, wobei Synergieeffekte zwischen den herangezogenen Abfällen genutzt werden sollen. Im Gegensatz zur separaten Aufarbeitung dieser Reststoffströme, ermöglicht die Kombination ein erhöhtes Ausbringen an Wertstoffen und die Etablierung von Verfahren in Regionen, in welchen bei ausschließlicher Verwendung eines Abfallstroms keine wirtschaftliche Aufarbeitung aufgrund der anfallenden Mengen möglich wäre. Durch die gezielte und sinnvolle $\mathrm{Zu}$ sammenführung und Aufarbeitung von komplexen, metallhaltigen Reststoffen wie z.B. Aktivmaterial aus Lithium-Ionen-Batterien und Müllverbrennungsaschen lassen sich flexible, aber dennoch selektive Prozesse zur Wertmetallrückgewinnung realisieren. Durch die Einsparung von langen Transportwegen und der Reduzierung des Energiebedarfs im Bereich der Erzaufbereitung und -verarbeitung können nachhaltige Methoden abgebildet und die Rohstoffverfügbarkeit durch die Erhöhung der Recyclingquote verbessert werden.

Schlüsselwörter Recycling

Metallhaltige Reststoffe

Müllverbrennungsasche

Aktivmaterial · Synergieeffekte

DI Dr. E. Gerold $(\bowtie)$.

Univ.-Prof. DI Dr. H. Antrekowitsch Lehrstuhl für Nichteisenmetallurgie, Montanuniversität Leoben, Franz Josef-Straße 18, 8700 Leoben, Österreich

eva.gerold@unileoben.ac.at
Potenzial assessment of synergy effects for the simultaneous recovery of valuable metals from complex, metal-containing residues

Abstract The aim of this work is the assessment of the possibilities for a simultaneous reprocessing of different, metal-containing residual waste streams from the industry, whereby synergy effects between the used wastes are to be consulted. In contrast to the separate reprocessing of these waste streams, the combination allows an increased yield of valuable materials and the establishment of processes in regions, where the exclusive application of one waste stream would not allow an economic reprocessing due to the accruing quantities. Through the targeted and sensible combination of complex, metal-containing residual materials such as active material from lithium-ion batteries and waste incineration ashes, flexible as well as selective processes for the recovery of valuable metals can be realized. By saving long transport routes and reducing energy requirements in the area of ore preparation and processing, sustainable methods can be mapped and raw material availability can be improved by increasing the recycling rate.

Keywords Recycling · Metal-containing residues . Waste incineration ash . Active material $\cdot$ Synergy effects

\section{Einleitung}

Die österreichische Recyclingwirtschaft steht in naher Zukunft, betreffend der immer wichtiger werdenden Aufarbeitung von metallhaltigen Reststoffen wie z.B. Lithium-Ionen-Batterien, vor einigen Herausforderungen. Zum einen hat sich bis heute kein Verfahren etabliert, das eine elementspezifische Rückgewinnung der einzelnen Bestandteile ermöglicht und zum anderen liegen die anfallenden Mengen in den nächsten Jahren in einem Bereich von unter $5000 \mathrm{t} / \mathrm{a}$, was eine singuläre wirtschaftliche Aufbereitung sehr schwierig macht. Um einen Export der Batterien und damit einer wichtigen Rohstoffquelle für kritische Elemente in andere Länder zu verhindern, gilt es daher Verfahren $\mathrm{zu}$ entwickeln, die eine gemeinsame Aufarbeitung mit anderen Reststoffen ermöglichen, die im optimalen Fall ebenfalls noch nicht ökonomisch sinnvoll recycelt werden können. $\mathrm{Zu}$ diesen Reststoffen zählen die Verbrennungsrückstände aus der kommunalen thermischen Abfallverwertung, die aktuell nach einer groben Metallentfrachtung bzw. Zerkleinerung deponiert werden. Diese Aufbereitung schließt allerdings nicht die Feinfraktion mit einer Korngröße kleiner $4 \mathrm{~mm}$ sowie die Kesselund Filteraschen ein, wodurch ein beträchtlicher Wertanteil dem Stoffkreislauf entzogen wird (Bundesministerium für Klimaschutz et al. 2020).

In der Schweiz ist seit 2021 eine Deponierung von Flugasche nur mehr nach Rückgewinnung der enthaltenen Metalle zulässig. Vorrangig kommt dafür die saure Flugaschewäsche (FLUWA) zum Einsatz, bei der mit Hilfe des sauren Waschwassers aus der Rauchgasreinigung die Schwermetalle aus der Asche extrahiert werden. In späteren hydrometallurgischen Gewinnungsverfahren können Metalle wie $\mathrm{Zn}, \mathrm{Pb}, \mathrm{Cu}$ und $\mathrm{Cd}$ anschließend zurückgewonnen werden. Nach diesem Vorbild wird am Lehrstuhl für Nichteisenmetallurgie untersucht, inwieweit eine Laugung der Flugasche sich mit jener anderer metallhaltiger Reststoffe kombinieren lässt. (Weibel et al. 2016).

Übergeordnetes Ziel dieser Untersuchungen ist eine simultane Aufarbeitung von verschiedenen, metallhaltigen Reststoffströmen aus der Industrie, wobei Synergieeffekte zwischen den herangezogenen Abfällen genutzt wer- 
den sollen. Durch eine Prozess- und Technologieentwicklung zur Verarbeitung von komplexen Rückständen mit schwankender Zusammensetzung im Bereich der Metallurgie sowie einer Erarbeitung von Verfahren zur Multimetallrückgewinnung aus primären und sekundären Quellen, kann eine Verbesserung der Rohstoffverfügbarkeit nachhaltig realisiert werden. Dieser Ansatz soll zu einer Schließung der Stoffkreisläufe führen und entspricht somit dem Gedanken der Kreislaufwirtschaft. Des Weiteren ermöglicht die simultane Aufarbeitung mehrerer Abfallströme einen Vergleich der angewandten Techniken für die entsprechenden Reststoffe und führt somit zu einem Wissensgewinn über die Möglichkeiten der Zusammenführung verschiedener Reststoffgruppen. Darüber hinaus können hochwertige Metalle und kritische Rohstoffe rückgewonnen und die Reststoffe einer stofflichen Verwertung im z.B. Bauwesen zugeführt werden. In diesem Zusammenhang ist die Vernetzung von aufbereitungstechnischen Operationen (Zerkleinerung, Sortierung und Agglomeration) mit den metallurgischen Prozessen von großer Bedeutung. Erst dadurch sind eine Trennung von hochwertigen Metallen und die gezielte Einstellung eines verwertbaren Rückstandes möglich. Im Rahmen der Arbeiten wird angestrebt, Müllverbrennungsaschen, Schlacken aus der metallurgischen Industrie und Materialien von der Lithium-IonenBatterie-Aufbereitung (Aktivmasse) gemeinsam zu recyceln sowie mögliche Verwertungswege synergetisch $\mathrm{zu}$ untersuchen. Daher werden die zu behandelten sekundären Rohstoffe näher betrachtet, um die Interaktion der notwendigen Technologien zu erläutern.

\section{Recyclingpotenzial von ausgewählten metallhaltigen Reststoffen}

Die jährlich anfallenden Mengen an Müllverbrennungsschlacken und -aschen belaufen sich für das Jahr 2018 auf rund 516.000t in Österreich, wobei ein großer Anteil dieser Reststoffe nach einer Ausstufung und Verfestigung einer Deponie zugeführt wird $(620.000 t$ an Schlacken und Aschen aus Abfallverbrennungsanlagen im Jahr 2018). Anhand dieser Angaben lässt sich das Verwertungspotenzial vor allem aufgrund der enormen anfallenden Mengen darstellen. Des Weiteren entstehen auch rund 122.000t an Flugaschen und -stäuben aus Abfallverbrennungsanlagen (Stand 2018), welche eine ähnliche Aufbereitung mit einer anschließenden Deponierung durchlaufen. Im Bereich der Lithium-Ionen-Batterien liegen die Mengen aktuell bei ca. $5000 \mathrm{t}$ im Jahr (Stand 2020), wobei in diesem abfallwirtschaftlichen Teilbereich ein starker Anstieg der Reststoffmassen in den nächsten Jahren erwartet wird. Je nach verfolgtem Szenario belaufen sich die anfallenden Mengen im Jahr 2030 auf 25.000 t und im Jahr 2035 bereits auf über $100.000 \mathrm{t}$ in Österreich. Im Rahmen dieser Abschätzung zeigt sich deutlich, dass eine Aufarbeitung dieser Reststoffströme vor allem aufgrund der stetig wachsenden Quantität ein zentrales Ziel der österreichischen Abfallwirtschaft darstellen muss. (Bundesministerium für Klimaschutz et al. 2020).

\section{Aktueller Stand der Technik im Bereich der Aufbereitung und des Recyclings von \\ Müllverbrennungsrückständen sowie verbrauchten Lithium- Ionen-Batterien}

Im Allgemeinen kann davon ausgegangen werden, dass sich durch die eingeführte Getrenntsammlung von Verpackungsabfällen, Glas, Papier und biogenen Abfällen der zu beseitigende Restmüll in seiner Zusammensetzung gegenüber dem Ende der achtziger Jahre anfallenden Müll deutlich verändert hat. Für die erforderliche Technik und Betriebsführung von Müllverbrennungsanlagen hat sich dadurch jedoch nur wenig geändert, da der Heizwert des Mülls weitgehend ident blieb. Müllverbrennungsschlacken und -aschen sind feste Rückstände, die bei der thermischen Behandlung von Siedlungsabfällen in Müllverbrennungsanlagen (MVA) entstehen. Die einzelnen Verbrennungslinien moderner Müllverbrennungsanlagen verfügen meist über mehrere unabhängig steuerbare Regeleinrichtungen, welche es ermöglichen, die brennbaren Bestandteile des Abfalls fast vollständig bei über $850^{\circ} \mathrm{C}$ zu verbrennen. Dies führt zu einer Gewichtsreduktion von 65 bis 75 Massenprozent, das heißt bei der Verbrennung von einer Tonne Haus- und Gewerbemüll fallen je nach Zusammensetzung des eingesetzten Siedlungsabfalls neben den Rückständen der Abgasreinigung, den Kesselaschen und Filterstäuben etwa
250 bis $350 \mathrm{~kg}$ Müllverbrennungs-Rohschlacke an. Diese Schlacke hat nach einer Durchlaufzeit von bis zu neunzig Minuten eine Temperatur von 500 bis $600^{\circ} \mathrm{C}$. Sie wird daher vor der sich anschließenden Entsorgung, gemeinsam mit dem Rostdurchfall, zunächst in einem Nassentschlacker abgekühlt (Schmidt und Weippert 2016; Breitenstein et al. 2015; Lück 2004; Kersten 1996).

Müllverbrennungsschlacke ist ein inhomogenes, partikelförmiges Stoffgemisch. Ihre Beschaffenheit und $\mathrm{Zu}-$ sammensetzung wird zum einen von der Abfallzusammensetzung und zum anderen von den Prozessbedingungen wie der Ofentemperatur, der Verweilzeit auf dem Rost und der Homogenisierung des Abfalls vor der Aufgabe auf den Rost bestimmt. Müllverbrennungsschlacken können über die Ausbrandparameter Glühverlust und TOC-Gehalt (Total Organic Carbon) charakterisiert werden. Diese sind ein Maß für die Vollständigkeit des vorangegangenen Verbrennungsvorgangs und indirekt auch für den Grad der Inertisierung des behandelten Abfalls sowie für die Zerstörung oder Einbindung von Schadstoffen in die Schlacke. Zusätzlich unterliegt die Schlacke nach ihrem Austrag komplexen Alterungsreaktionen. Dabei handelt es sich im Wesentlichen um Mineralneu- und -umbildungen, bei denen zunächst noch verfügbare Inhaltsstoffe weitgehend inertisiert werden und die spezifische Oberfläche zunimmt. Die Massenanteile der Inhaltsstoffe variieren je nach Verbrennungstechnik, -temperatur und Abfallzusammensetzung. Als Anhaltsgröße lässt sich folgende durchschnittliche Schlackenzusammensetzung angeben: (Schmidt Weippert 2016; Breitenstein et al. 2015; König 1993)

- Mineralische Fraktion: 85 bis 90 Massen-\%,

- Unverbranntes: 1 bis 5 Massen-\%,

- Metallschrott: 7 bis 10 Massen-\%.

Die mengenmäßig bedeutendste Gruppe ist die mineralische Fraktion. Diese besteht neben Schlackenstücken und Asche auch aus Bestandteilen wie Beton, Ziegeln, Steinen und Glas. Laut Literaturangaben sind etwa $40 \%$ dieser mineralischen Schlackenanteile amorph (Gläser, Keramik, Aschen) und $60 \%$ kristallin (neu gebildete Minerale). Nicht oder nur teilweise verbranntes Material wird in der Stoffgruppe „Unverbranntes“ zusammengefasst. 
In dieser finden sich Papier-, Holz-, Kunststoff- und Textilreste wieder. Der Metallschrott besteht aus Nichteisenund Eisenmetallen. Weißblech (z.B. Dosenschrott), Aluminium und seine Legierungen, Kupfer, Messing, Metalle in Ölfiltern usw. sind die wesentlichen Komponenten dieser Stoffgruppe. Die thermischen Bedingungen während der Müllverbrennung führen zu einem vollständigen oder teilweisen Schmelzen und auch zur Oxidation der metallischen Komponenten. Durch Kontakt angeschmolzener Schrottstücke mit anderen Partikeln entstehen Agglomerate mit ausgeprägten Verwachsungseigenschaften. Dabei handelt es sich überwiegend um oberflächliche Verwachsungen mit mineralischen Komponenten, die durch eine moderate mechanische Kornbeanspruchung wieder aufgelöst werden können (Schmidt und Weippert 2016; Breitenstein et al. 2015; Lück 2004; Kersten 1996; König 1993).

Neben diesen Hauptbestandteilen sind in Müllverbrennungsschlacken Alkali- und Erdalkaliverbindungen, Chloride $(>1 \mathrm{~g} / \mathrm{kg})$, Fluoride $(>1 \mathrm{~g} / \mathrm{kg})$, Sulfate sowie unterschiedliche schwerflüchtige Schwermetalle und deren Verbindungen enthalten. In Abhängigkeit von den Verbrennungsbedingungen und dem jeweiligen Dampfdruck der Schwermetalle reichern sich diese in der Schlacke in unterschiedlichen Konzentrationen an. Die Belastung mit Dioxinen und Furanen fällt bei Schlacken im Vergleich zu den übrigen Rückständen aus der Müllverbrennung (z. B. Filterstäube) deutlich geringer aus. (Breitenstein et al. 2015; Kersten 1996).

Die Aschen und Schmelzprodukte unterscheiden sich sowohl morphologisch als auch in der Korngröße. Die Aschen werden aus Glasabrieb, anorganischen und organischen Rückständen sowie Ruß- und Staubpartikeln mit einer durchschnittlichen Größe von 0,002 bis $2 \mathrm{~mm}$ gebildet. Demgegenüber stellen die Schmelzprodukte stark poröse, unregelmäßig geformte Mineralkörner einer Partikelgröße von größer $2 \mathrm{~mm}$ dar, die aus einer silikatischen Matrix (Glas) mit kristallinen Neubildungen (Silikate und Oxide) entstehen. (Schmidt und Weippert 2016).

Die Verwertung von Müllverbrennungs-Rohschlacken im Tiefbau ist wegen der dort möglichen Auswaschung von Salzen und Schwermetallen in der zunächst vorliegenden, unbehandelten Form, das heißt ohne weitere Vorbe- handlung, ökologisch inakzeptabel. Im Unterschied zur Deponie kann das Sickerwasser hier nicht kontrolliert gesammelt und behandelt werden, sondern gelangt diffus in den Boden und gegebenenfalls in das Grundwasser. Daher erfordert die Verwertung von Schlacke eine weitere Reduzierung eluierbarer Bestandteile durch eine geeignete Aufbereitung. Zielsetzung der Schlackenaufbereitung ist es, absatzfähige, das heißt marktfähige, umweltgerechte Produkte herzustellen. Entscheidend für einen wirtschaftlichen Betrieb der Aufbereitungsanlage ist das Erreichen der Qualitätsanforderungen der jeweiligen Abnehmer, Bauträger, Aufsichtsbehörden und des Gesetzgebers. Im Rahmen von gesetzlichen Regulierungen werden Anforderungen an charakteristische Stoffparameter, Güteüberwachungskriterien, Anforderungen an den Einsatz von Schlacke im Straßenbau usw. definiert. Für eine Verwertung der Müllverbrennungsschlacken sollten zur Qualitätssicherung zumindest die Rückstände separiert, die Eisenfraktion und andere Metalle abgetrennt und die Schlacken klassiert werden. Eine umfassende Verwertung und gezielte Abtrennung von Wertstoffen, um eine hochwertige stoffliche Verwertung der Mineralfraktion zu garantieren, ist zurzeit nicht gegeben (Schmidt und Weippert 2016; Breitenstein et al. 2015; Lück 2004; Kersten 1996).

Für die Aufbereitung von Müllverbrennungsschlacke hat sich aufgrund der Veränderung der Einsatzstoffe und der getrennten Sammlung in den letzten Jahren die Menge an aussortierbarem Eisenschrott annähernd halbiert. Die einfachste Form der Müllverbrennungsschlackenaufbereitung ist die Schrottsortierung mit Überbandmagnetscheidern, die oft schon bereits nach dem Nassentschlacker installiert sind. Der Eisengehalt des so erzeugten Eisen- und Stahlgemischs beträgt - je nach Art des Verbrennungssystems lediglich 50 bis 60 Massen-\%. Die Verunreinigungen bestehen aus an den Metallteilen fest angebackenen Schlacke- und Aschebestandteilen. Dieses Material muss daher vor seiner metallurgischen Verwertung zunächst durch Schreddern in ein stahlwerksfähiges Endprodukt mit einem Eisengehalt von etwa 95 Massen-\% und einer Schüttdichte von rund $1 \mathrm{t} / \mathrm{m}^{3}$ umgewandelt werden. In den meisten Aufbereitungsanlagen wird Müllverbrennungsschlacke nach einer Lagerung, die je nach
Lagerbedingungen zwischen zwei Wochen und drei Monaten liegt, trocken klassiert und sortiert. In vielen Fällen wird daher auch nicht ausreichend gealtertes Material aufbereitet. Das Ziel der meist mehrstufigen Klassierung ist es Kornklassen $\mathrm{zu}$ erzeugen, die im Tiefbau für jeweils definierte Einsatzzwecke benötigt werden. Derartige Anlagen bestehen in der Regel aus nicht mehr als drei oder vier Sieben, zwei Magnetscheidern sowie einer Reihe von Förderbändern. Unverbrannte Materialien und Nichteisenmetalle werden in manchen Anlagen durch Handsortierung abgetrennt. Es ist offensichtlich, dass mit dieser einfachen Aufbereitungstechnik lediglich gröbere Bestandteile erfasst werden können und die erreichten Ausbringungsquoten sehr niedrig sind. Die nicht erfassten Komponenten bleiben im Gutstrom und führen so nicht nur zu einer visuellen Beeinträchtigung, sondern auch zu ungünstigen chemisch-physikalischen Eigenschaften des mineralischen Produkts. Die Verwertungsmöglichkeiten dieser einfach aufbereiteten Produkte sind daher auf wenige Einsatzgebiete begrenzt. Waschverfahren zur Abscheidung von wasserlöslichen Inhaltsstoffen wie Chloriden oder Sulfaten wurden in den achtziger Jahren im Laborund Pilotmaßstab erprobt. Die Versuche zeigten deutlich, dass so eine nennenswerte Schadstoffabreicherung erreicht werden kann. Es ist offensichtlich, dass die industrielle Anwendung dieses Verfahrens aufgrund der damit verbundenen Aufwendungen für die Wasserbewirtschaftung zu hohen Kosten führt (Schmidt und Weippert 2016; Breitenstein et al. 2015; Lück 2004; Kersten 1996; König 1993).

Die Zwischenprodukte beim Recycling von Batterien stellen ebenfalls einen interessanten Produktstrom dar, welcher aufgrund des Metallanteils sinnvoll einer gemeinsamen Verwertung mit Schlacken und Aschen aus der metallurgischen Industrie und Müllverbrennung zum Einsatz kommen kann. In diesem Zusammenhang ist im Speziellen eine geeignete Aufbereitung zu entwickeln, um vor einem metallurgischen Schritt mögliche Schadstoffe oder hochwertige Metalle $(\mathrm{Al}, \mathrm{Cu})$ gezielter abzutrennen. Für die verschiedenen Batteriesysteme mit ihren sehr unterschiedlichen Komponenten (Metalle, Oxide, Elektrolyte, Kunststoffe) und Problemstoffen ist kein einheitliches Recyclingverfahren verfügbar. Ein 
solcher Prozess könnte aufgrund der stark differenzierten Ausgangsmaterialien auch nicht das Recycling der verschiedenen Stoffe mit großer Ausbeute in hoher Endqualität gewährleisten. Die anfallenden Altbatterien müssen deshalb vor den nachfolgenden Behandlungsschritten in die verschiedenen Batteriesysteme sortiert werden. Diese Sortierprozesse lassen sich für Starterbatterien (Blei-Säure-Batterien) und auch für Industriebatterien relativ gut realisieren. Problematisch ist die Identifizierung der relativ kleinen Gerätebatterien (Rundzellen, Blockbatterien, Knopfzellen) und der Batterie-Packs aus mehreren Einzelzellen $\mathrm{Xu}$ et al. 2008; Velázquez-Martínez et al. 2019; Zheng et al. 2018; Korthauer 2013).

Für Kleingerätebatterien bestehen bereits seit Jahren entsprechende Recyclinganlagen. Grundsätzlich haben sich vor allem hydro- und/oder pyrometallurgische Verfahren durchgesetzt. Dabei ist es wichtig, zwischen dem eigentlichen Recycling und dem Refining zu unterscheiden. Nicht alle Recyclingunternehmen führen auch die RefiningSchritte durch, aus denen letztlich die Wertmaterialien in aufbereiteter Form für die Industrieanwendungen zurückgewonnen werden. Das pyrometallurgische Verfahren kann speziell auf eine hohe Rückgewinnungsquote für Nickel und Kobalt (Nebenprodukt Kupfer) ausgelegt werden. Es ist aber auch für das Recycling von anderen Ni-Co-haltigen Materialien, wie z. B. verbrauchte Katalysatoren, einsetzbar. Sollte es aufgrund der Marktsituation interessant werden Lithium als Element, in oxidischer Form oder als Lithiumkarbonat zurückzugewinnen, so stehen bereits die benötigten Prozesskonzepte im Labor- und Pilotmaßstab zur Verfügung. (Korthauer 2013).

Im Gegensatz zu Batterien aus portablen Anwendungen erfordern die erheblich größeren aus Fahrzeugen mit vollelektrischem Antrieb (EV) oder Hybridantrieb (HEV) aus prozesstechnischen Gründen spezielle Vorbereitungsschritte. Neben der mechanischen Behandlung, die aufgrund der Abmessungen, des Gewichtes (50 bis $450 \mathrm{~kg}$ ) und der Materialvielfalt notwendig ist, müssen wegen der in den Batterien gespeicherten Restenergien und in Verbindung mit dem jeweiligen mechanischen bzw. elektrochemischen Zustand der Batterien zusätzliche Maßnahmen erfolgen, um Gefährdungen bei der Lagerung, dem Transport und der Handhabung auszuschließen. Eine sehr einfache Sortierung kann nach der Größe durch Siebung erfolgen (Abtrennung von Knopfzellen). Die Zink-KohleBatterien sind mittels eines Elektromagneten abzutrennen, da sie im Unterschied zu allen anderen Typen keinen Eisenmantel besitzen. Rundbatterien lassen sich von anders geformten Systemen mittels eines Drehtellers trennen. Anschließende Prozesse differenzieren zwischen visueller bzw. manueller und automatischer Sortierung. Bei der manuellen Variante müssen die anfallenden Batterie-Packs händisch aussortiert werden, um anschließend das Kunststoffgehäuse zu entfernen. Im Bereich der automatisierten Prozesse erfolgt die Sortierung der Einzelzellen mittels sensorgestützter Sortiertechnik unter Verwendung verschiedener Sensorsysteme. Dazu zählt die Wägung in Kombination mit einem elektromagnetischen Sensor, der die verschiedenen WerkstoffSysteme magnetischer Rundzellen aufgrund unterschiedlicher Störungen des Magnetfeldes erkennt. Diese Identifikation steuert den Auswurfmechanismus. Des Weiteren erfolgt der Einsatz von Röntgenverfahren, um nach einer Größensortierung das Batteriesystem aufgrund der erkannten Röntgenstrahlung zu identifizieren und zu sortieren. Eine zusätzliche Sortierung nach Hg-freien Batterien kann durch Erkennung eines aufgebrachten UV-sensiblen LackPigments erreicht werden. Auf Basis dieser bereits etablierten Grundoperationen soll eine Evaluierung und Weiterentwicklung am Lehrstuhl für Nichteisenmetallurgie stattfinden, um Prozesse auch synergetisch für weitere eingesetzte Reststoffe zu nützen. Der Einsatz von modernster Sensortechnik ermöglicht eine Adaptierung und Verbesserung der bisher eingesetzten Systeme, um einen flexiblen Prozess für unterschiedliche Einsatzströme zu generieren. Dieser Verfahrensschritt stellt eine essentielle Grundoperation im Rahmen dieses Forschungsbereichs dar, da alle anschließenden Technologien auf dieser Stufe aufbauen (Xu et al. 2008; Velázquez-Martínez et al. 2019; Zheng et al. 2018; Korthauer 2013).

Die industrielle Verwertung von $\mathrm{Li}$ thium-Ionen-Batterien findet zurzeit hauptsächlich für die Systeme der ersten und zweiten Generation statt. Relevante Massenströme gibt es bislang hauptsächlich aus dem Bereich der Gerätebatterien, wobei der Anfall an Altbatterien bei der Elektromobilität stetig zunimmt. Verfahrenserweiterungen für Lithium-Ionen-Traktionsbatterien der Elektromobilität wurden zum Teil bereits entwickelt und stellen derzeit ein breites Themengebiet in der Forschung dar. Unterschiedliche Routen werden industriell genutzt, die allerdings bisher hauptsächlich auf die Rückgewinnung von Kobalt und Nickel ausgelegt sind. Unedle Metalle wie Lithium, Aluminium, Mangan usw. sammeln sich meist in der silikatischen Schlacke des Schmelzprozesses, während Halogene und ggfs. vorhandene bzw. mitgeschleppte leicht-flüchtige Metalle in die Stäube ausgetragen werden. Die Batterien werden in einer ersten Behandlungsstufe vom Stahlgehäuse befreit und zusammen mit anderen $\mathrm{Ni}$ Co-haltigen Abfällen und Zuschlägen in Schmelzbadreaktoren reduzierend geschmolzen. Der Elektrolyt, die Polymere und der Graphit verbrennen dabei und dienen auch als Reduktionsmittel. Metallschmelzphase und Schlackenphase werden granuliert. Die Metallgranalien werden hydrometallurgisch zu Nickel-, Kupfer- und Kobalt-Metallen und Verbindungen verarbeitet. Bei alleinigem Einsatz von Lithium-Ionen-Batterien entsteht eine lithium-haltige Schlacke, die gezielt als Zementzuschlagstoff (Lithium erhöht die Abbindegeschwindigkeit bestimmter Zemente) genutzt werden kann. Obwohl Umicore der weltgrößte Verwerter von Lithium-Ionen-Batterien ist, reichen die derzeit anfallenden Mengen alleine noch nicht für eine wirtschaftliche Umsetzung dieses Prozessschrittes aus. Bereits in diesem Zusammenhang zeigen sich die möglichen Interaktionen bei der Verwertung unterschiedlicher Fraktionen. Bei den aktuellen Steigerungsraten im Verbrauch von Lithium-Ionen-Gerätebatterien und dem Beginn des Aufkommens erster Altakkumulatoren aus dem Bereich der Elektromobilität kann sich dieses in absehbarer Zeit ändern (Zheng et al. 2018; Korthauer 2013).

Grundlegend für alle nachgeschalteten Prozesse stellt die optimale Aufbereitung der Lithium-Ionen-Altbatterien eine Grundvoraussetzung dar, insbesondere dann, wenn eine synergetische Verwertung mit anderen Materialien (metallurgische Schlacken und Müllverbrennungsrückständen) erfolgen soll. Aufgrund der Selektivität der derzeitigen Aufbereitungsverfahren ergeben sich Grenzen in den Recyclingprozessen, da nur bestimmte Wertstoffe wie Kupfer, Kobalt oder Nickel zurückge- 

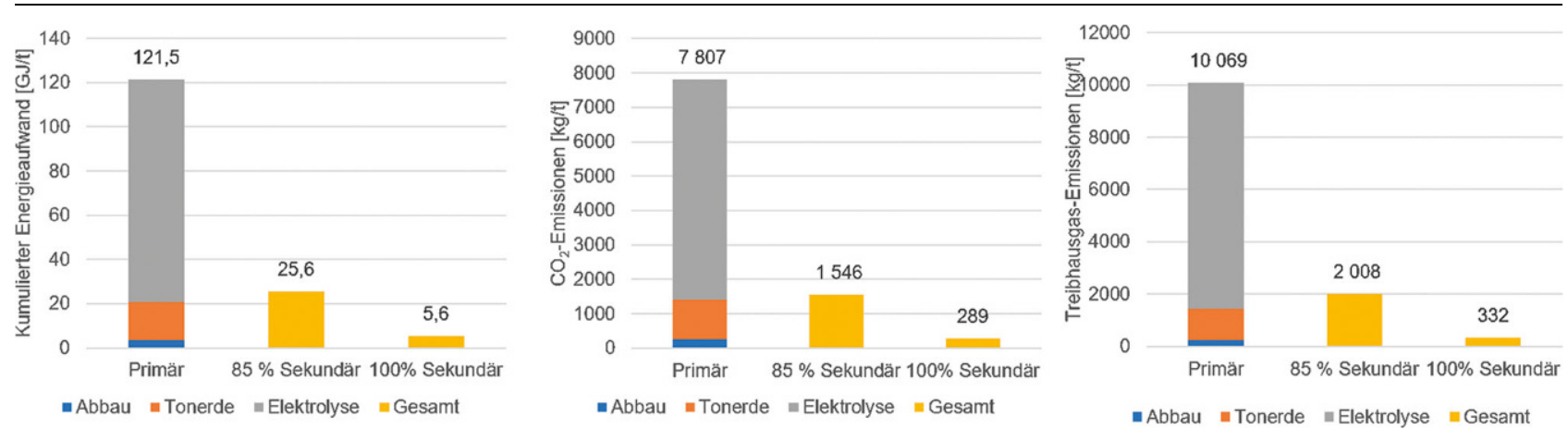

Abb. 1 Aufstellung des kumulierten Energieaufwands, der $\mathrm{CO}_{2}$ - und Treibhausgasemissionen für die primäre und sekundäre Herstellung von Aluminium (Frischenschlager et al. 2010)

wonnen werden. Die stoffliche Zusammensetzung, die Gehalte sowie das Recyclingpotenzial der einzelnen in der Batteriezelle enthaltenen Komponenten liegen in folgenden Bereichen: $(\mathrm{Xu}$ et al. 2008; Velázquez-Martínez et al. 2019; Zheng et al. 2018; Korthauer 2013):

- Aluminium, Cr-Ni-Stahl aus dem Gehäuse: 14 bis 24 Massen-\%,

- Kupfer aus den Anodenfolien: 8 bis 10 Massen-\%,

- Graphit und Binder aus der Anodenbeschichtung: 16 bis 20 Massen-\%,

- Aluminium aus der Kathodenfolie: 3 bis 4 Massen-\%,

- Aktivmaterial, Binder und Ruß aus der Kathodenbeschichtung: 30 bis 35 Massen-\%,

- Organische Lösungsmittel, Leitsalz und Additive aus dem Elektrolyt: 10 bis 15 Massen-\%,

- Polyethylen aus der Separatorfolie: 6 bis 10 Massen-\%.

Die chemisch aktiven Substanzen, die für die Energiespeicherung in Batterien verantwortlich sind, stellen das Kernstück der Elektroden dar und werden als Aktivmaterial bezeichnet. Um den elektrischen Strom optimal zu leiten und somit die benötigten Leistungsdaten zu erzielen, erfolgt die Aufbringung dieser Aktivmaterialien auf Stromkollektoren. Diese bestehen in klassischen Lithium-Ionen-Batterien aus Kupferund Aluminiumfolien. Prinzipiell ist die Zusammensetzung von Lithium-IonenBatterien und auch von der verwendeten Aktivmasse stark vom Hersteller und Batterietyp abhängig. Je nach Art des Kathodenmaterials enthält eine klassische LIB zwischen 5-20\% Kobalt, 5-10\% Nickel und 5-7\% Lithium. Des Weiteren fallen auch organische Chemikalien aus dem Elektrolyten und Binder, Folienbestandteile, Kunststof- fe von den Separatoren, Graphit und die Stahlgehäuse als Reststofffraktionen an (Xu et al. 2008; Velázquez-Martínez et al. 2019; Zheng et al. 2018; Korthauer 2013).

\section{Rückgewinnungs- und Einsparungspotenzial für ausgewählte Metalle}

Bei der Betrachtung der zu behandelnden Materialien lassen sich deutliche Überschneidungen in den $\mathrm{Zu}$ sammensetzungen der Schwarzmasse aus Lithium-Ionen-Batterien und der Müllverbrennungsschlacke bzw. -asche sowie von metallurgischen Schlacken erkennen. Vor allem die Wertmetallgehalte an Kupfer und Aluminium sowie kritische Metalle sollen in diesem $\mathrm{Zu}$ sammenhang Beachtung finden. Durch eine gemeinsame Aufbereitung können somit nicht nur der Wertmetallgehalt im erzeugten Produkt erhöht, sondern auch Synergieeffekte genutzt werden.

Bei Betrachtung des Wertstoffs Aluminium lässt sich hier beispielsweise ein besonders hohes Potenzial im Bereich der Energieeinsparung darstellen. Der Vergleich der Primär- und Sekundärherstellung von Al zeigt, dass bei der Sekundärproduktion von Aluminium ca. $96 \mathrm{GJ} / \mathrm{t}$ des kumulierten Energieaufwands $(79 \%)$, ca. $6262 \mathrm{~kg} / \mathrm{t}$ der $\mathrm{CO}_{2}$-Emissionen $(80 \%)$ und rund $8100 \mathrm{~kg} / \mathrm{t}$ der THG-Emissionen $(80 \%)$, bezogen auf die Primärherstellung mit österreichischem Strommix, vermieden werden. Bei der Produktion von Sekundäraluminium ohne Primärmetall-Zusatz kommt es zu einer Einsparung von $95 \%$ beim kumulierten Energieauswand (KEA) sowie von $97 \%$ bei den Treibhausgasemissionen (Frischenschlager et al. 2010; Reuter et al. 2019).
Die entsprechenden Einsparungspotenziale für Aluminium lassen sich Abb. 1 entnehmen und sind dort für den österreichischen Strommix zusammengefasst (Berechnungen für die Sekundärproduktion mit Mittelwerten über den Closed-Well- und Drehtrommelofen bei Schrotteinsatz) (Frischenschlager et al. 2010; Reuter et al. 2019).

Ähnliches lässt sich auch für die Rückgewinnung von Kupfer aus Sekundärmaterialien schlussfolgern, wobei in diesem Bereich zusätzlich auch eine Vermeidung von $\mathrm{SO}_{2}$-Emissionen realisiert werden kann. Durch die im Vergleich zu den primär gewonnenen Erzen ca. 30-fach höheren Gehalte an Kupfer in den anfallenden Schrotten und Sekundärrohstoffen, lässt sich ein deutlich effizienterer Prozess im Bereich des Recyclings von Kupfer realisieren. Zusätzlich kann durch entsprechende metallurgische Raffinationsschritte ein zum primär erzeugten Kupfer vergleichbares Produkt aus den anfallenden Abfällen gewonnen werden. Darüber hinaus ist durch das Recycling dieses Massenmetalls aus den angeführten Reststoffen auch die Rückgewinnung von kritischen Elementen möglich, welche sich häufig in der Metallphase (vor allem Kupfer) sammeln. Der Vergleich zeigt, dass bei der Sekundärproduktion rd. $46 \mathrm{GJ} / \mathrm{t}$ Energieverbrauch $(71 \%), 4460 \mathrm{~kg} / \mathrm{t}$ der $\mathrm{CO}_{2}$-Emissionen $(87 \%)$ und $4747 \mathrm{~kg} / \mathrm{t}$ der THGEmissionen (85\%) im Vergleich zur Primärherstellung vermieden werden. Die für das Wertmetall Kupfer beschriebenen möglichen Einsparungspotenziale sind in Abb. 2 dargestellt. (Korthauer 2013).

Des Weiteren zeigt sich beim Recycling von Lithium-Ionen-Batterien vor allem immer wieder in kleineren Ländern wie Österreich, mit einer begrenzten Produktion an Schwarzmasse, 

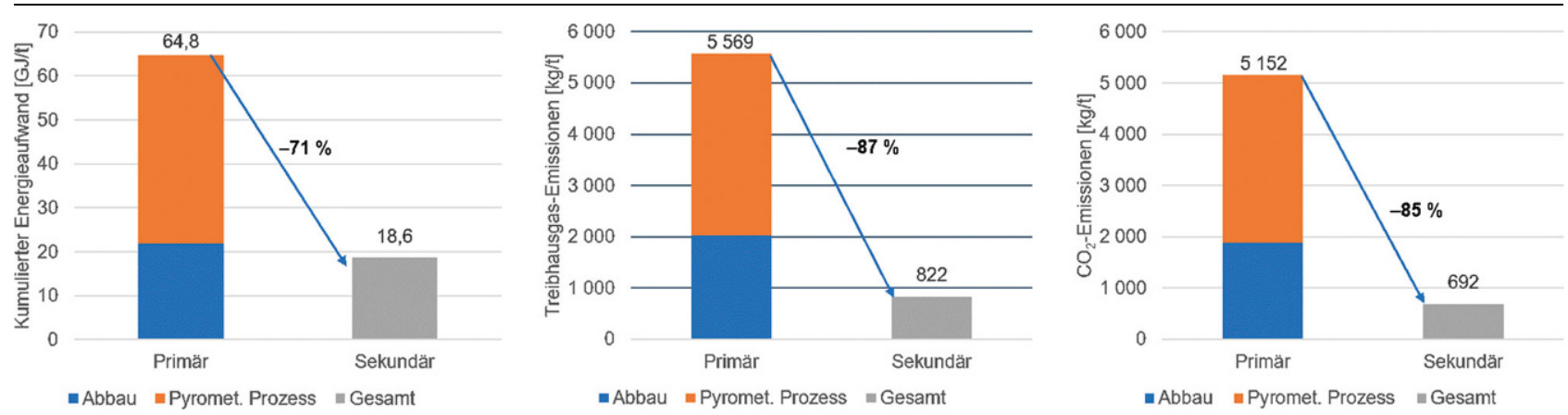

Abb. 2 Aufstellung des kumulierten Energieaufwands, der $\mathrm{CO}_{2}$ - und Treibhausgasemissionen für die primäre und sekundäre Herstellung von Kupfer (Frischenschlager et al. 2010)

dass eine singuläre Aufarbeitungsstrategie für diesen Reststoff sich kaum wirtschaftlich betreiben lässt. Viele österreichische Unternehmen weichen daher in ausländische Anlagen oder Standorte aus, um ausreichend Input-Material für die $\mathrm{zu}$ betreibende Aggregate bereitzustellen. Durch die Kombination unterschiedlicher Reststoffströme unter Beachtung derer Zusammensetzungen und Wertmetallgehalte lässt sich aber auch in Österreich und anderen kleineren Ländern eine Aufarbeitungsanlage betreiben.

Ein weiteres Ziel dieser Forschungsarbeit ist neben der Charakterisierung der einzelnen Reststoffströme und der Identifikation der Wertmetallgehalte vor allem die Erstellung einer kombinatorischen Matrix für die Verwertung unterschiedlicher Abfälle. Dabei sind die mengenmäßig großen Abfallströme, wie die Müllverbrennungsschlacke und -aschen, die Basis für das zu entwickelnde Verfahren. Dieses soll jedoch so flexibel gestaltet werden, dass weitere metallhaltige Reststoffe, wie zum Beispiel Aktivmasse von Lithium-Ionen-Batterien oder Schlacken aus der metallurgischen Industrie, mitbehandelt werden können. Auf Basis dieses Modells lässt sich ein neuer Prozess kreieren, welcher die Möglichkeit zur Aufarbeitung von unterschiedlichen metallhaltigen Reststoffströmen bietet, wodurch die Anwendbarkeit deutlich gesteigert wird.

\section{Hydrometallurgische} Rückgewinnung von Wertstoffen aus metallhaltigen Reststoffen

Die Überführung der Übergangsmetallverbindungen in meist anorganische Lösungen ermöglicht die Vorbereitung für die späteren Trennstufen. In diesem Zusammenhang stehen vor allem die Kriterien Selektivität und Effizienz des Prozesses im Vordergrund. Generell spielen beispielsweise im Rahmen des hydrometallurgischen Recyclings von Lithium-Ionen-Batterien klassische, anorganische Säuren wie z.B. $\mathrm{H}_{2} \mathrm{SO}_{4}, \mathrm{HNO}_{3}$ und $\mathrm{HCl}$ eine große Rolle, wobei häufig der Zusatz eines Reduktionsmittels (z.B. Wasserstoffperoxid) notwendig ist, um einen zufriedenstellenden Aufschluss zu erreichen. Dieser Effekt zeigt sich vor allem in der deutlich verbesserten Laugungseffizienz von Kobalt und Lithium im Bereich des Recyclings von LithiumIonen-Batterien, wobei ersteres durch die Reduktion von $\mathrm{Co}^{3+} \mathrm{zu} \mathrm{Co}^{2+}$ wesentlich leichter in Lösung geht. Neben den Chemikalien selbst spielen im Gebiet der Laugung die Parameter Temperatur, Dauer, Feststoff-Flüssigkeits-Verhältnis und Säurestärke eine wesentliche Rolle. In vielen Literaturstellen ist die Beschreibung von definierten Parameterkombinationen $\mathrm{zu}$ finden, wobei meist nur auf die Laugung eines spezifischen Kathodenmaterials eingegangen wird. Generell lässt sich zusammenfassen, dass erhöhte Temperaturen $\left(60-80^{\circ} \mathrm{C}\right)$ und niedrige Feststoff-Flüssigkeits-Verhältnisse $\left(20-50 \mathrm{~g} \cdot \mathrm{l}^{-1}\right) \mathrm{zu}$ hocheffizienten Prozessen führen. Allerdings muss in diesem Stadium auch bereits die Wirtschaftlichkeit solcher Prozesse in der Industrie bedacht werden, welche mit einem dementsprechend niedrigen Feststoffgehalt kaum zu realisieren ist. Bei Betrachtung der notwendigen Reaktionszeiten lässt sich in der Literatur ein relativ breites Spektrum finden, wobei die Gesamtdauer der Verfahren meisten zwischen ein und vier Stunden liegt. Häufig findet zusätzlich eine Ultraschallunterstützung der Methode statt, um den Aufschluss der festen Bestandteile zu verbessern ( $\mathrm{Li}$ et al. 2013, 2009, 2009, 2014; Lee Rhee 2002).

\section{Forschungsfortschritt am Lehrstuhl für Nichteisenmetallurgie}

Im konkreten Fall wird die Laugung an den am Lehrstuhl für Nichteisenmetallurgie der Montanuniversität Leoben entwickelten SeLiReco-Prozess (Selective Lithium Recovery) angelehnt, der die Aufarbeitung inkl. selektiver Metallrückgewinnung von Lithium-IonenBatterien ermöglicht. Dieser Prozess setzt sich prinzipiell aus mehreren Einzelstufen zusammen, wobei zu Beginn die schwefelsaure Laugung der aus der mechanischen Aufbereitung zurückgewonnenen Pulverfraktion einschließlich des Aktivmaterials steht (siehe Abb. 3). (Gerold 2021).

Die Laugung von Aktivmaterialien aus Lithium-Ionen-Batterien setzte ausgedehnte Vorarbeiten zur Einschränkung der Zielkenngrößen voraus. Als potenzielle anorganische Laugungsmedien kamen dabei vor allem Schwefelund Salzsäure aufgrund ihrer weiten Verbreitung und dem entsprechen wirtschaftlichen Potenzial in Frage. Wie bereits erwähnt, ermöglicht der gezielte Zusatz von $\mathrm{H}_{2} \mathrm{O}_{2}$ eine Verbesserung der Laugungseffizienz und kam daher auch in diesem Zusammenhang zum Einsatz. Zur Bestimmung eines optimalen Laugungsgebietes für Aktivmaterialen aus verbrauchten Lithium-Ionen-Batterien erfolgte die Durchführung von mehreren Experimentalreihen, welche auf Basis einer statistischen Versuchsplanung mit einem vollfaktoriellen Modell (inkl. Zentrumsversuche) geplant und ausgewertet wurden. Im Rahmen der durchgeführten Evaluierungen zeigte sich, dass hohe Temperaturen $\left(80^{\circ} \mathrm{C}\right)$ und die Verwendung von 2-molerer $\mathrm{H}_{2} \mathrm{SO}_{4}$ bei einem Feststoff-Flüssigkeitsverhältnis von $100 \mathrm{~g} \cdot \mathrm{l}^{-1}$ und einer Laugungsdauer von ca. vier Stunden zu 


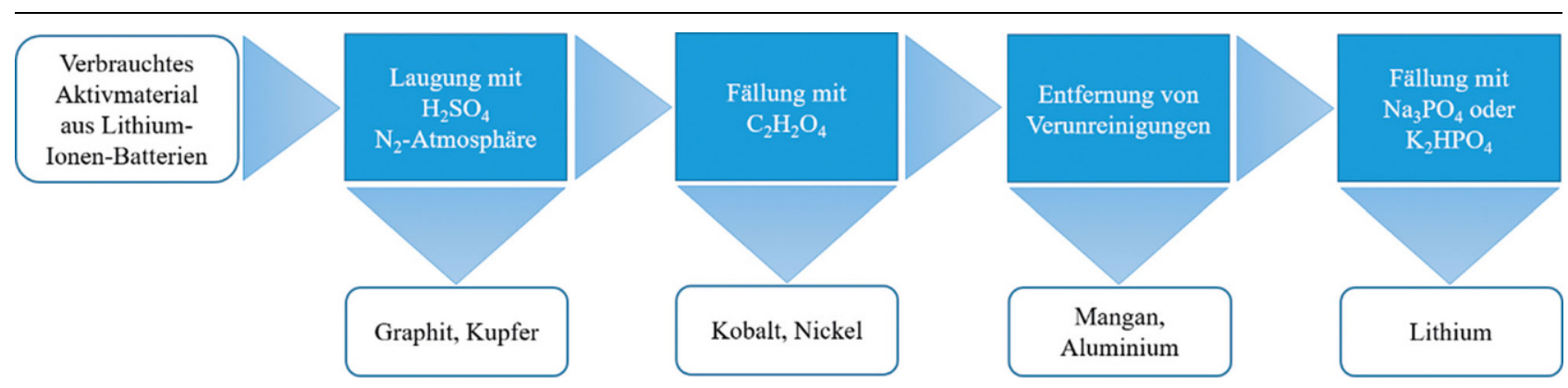

Abb. 3 Verfahrensschema des SeLiReco-Prozesses für das hydrometallurgische Recycling von verbrauchten Lithium-lonen-Batterien (Gerold 2021)

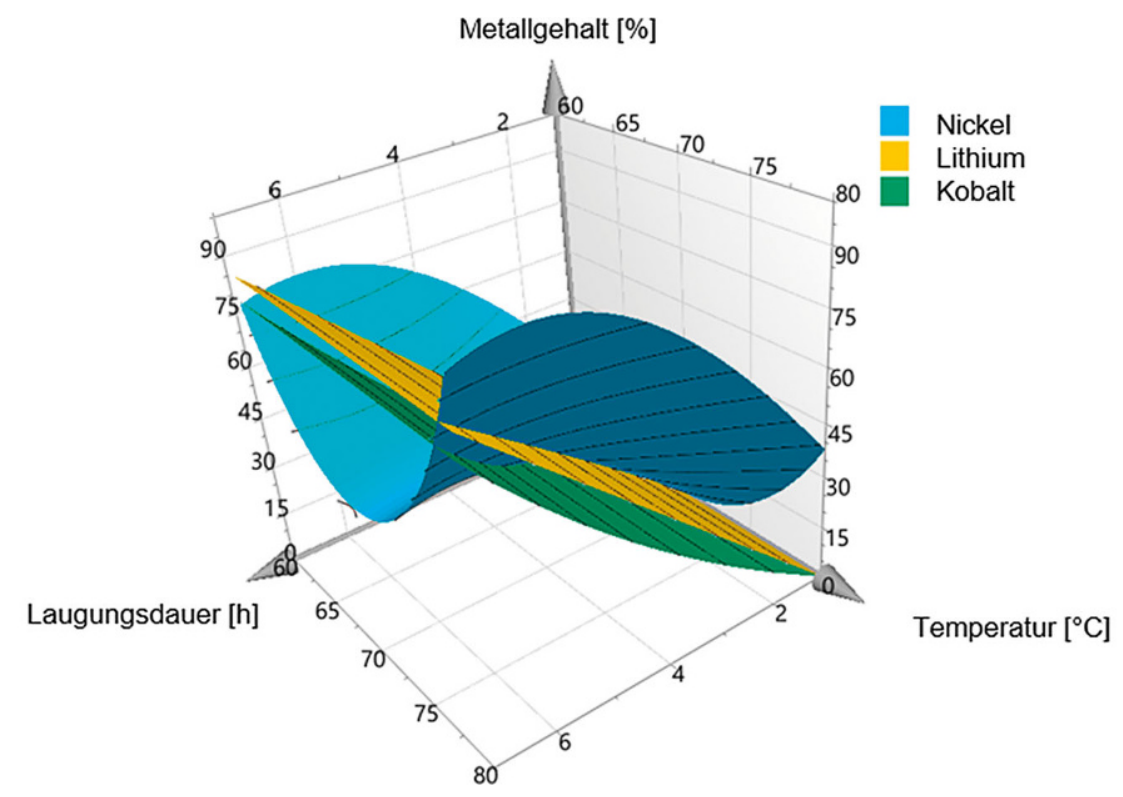

Abb. 4 Dreidimensionale Darstellung des Modells für die Laugung von Kobalt, Nickel und Lithium aus Aktivmaterial in Abhängigkeit der Haupteinflussfaktoren Laugungsdauer und Temperatur (Skalierung der Applikatenachse zwischen 0-100 für den Gehalt an Nickel, Kobalt und Lithium) (Gerold 2021)

optimalen Ergebnissen führen. Dies lässt sich auch in der statistischen Auswertung der durchgeführten Versuche in Abb. 4 erkennen. Bei Betrachtung der dem jeweiligen Element zugehörigen Laugungseffizienz zeigte sich im Rahmen dieser Experimentalreihe, dass sich diese für Kobalt und Nickel auf 95 bzw. $90 \%$ im Parameterbereich des optimalen Laugungspunktes beläuft. Lithium ließ sich aufgrund seiner guten Auslaugfähigkeit in Lösung überführen, wodurch eine nahezu vollständige Laugung der wichtigsten Wertmetalle erzielt werden konnte (Gerold 2021; Gerold et al. 2019a, 2019b).

Auf Basis dieser Ergebnisse wäre auch die Versuchsplanung und -durchführung für die Rückgewinnung von Metallanteilen aus MV-Aschen umzusetzen. Da sich im Rahmen der durch- an die optimierten Parameter fanden Laugungsexperimente statt, wobei die Variation der Säurekonzentration zwischen 0,5 und $2 \mathrm{~mol} / \mathrm{l}$ fixiert wurde. Das Feststoff-Flüssigkeitsverhältnis belief sich auf 50 bis $100 \mathrm{~g} / \mathrm{l}$ bei einer Temperatur zwischen 60 und $80^{\circ} \mathrm{C}$. Die Maximaldosierung an Oxidationsmittel $\left(\mathrm{H}_{2} \mathrm{O}_{2}\right)$ wurde mit 5Vol.-\% der Lösung fixiert, wobei ebenfalls Experimente ohne Wasserstoffperoxid erfolgten. Der zu untersuchende Feststoff verblieb zwischen 60 und $240 \mathrm{~min}$ in der Laugungslösung, um eine eventuelle Zeitabhängigkeit der Reaktion zu detektieren. Die Versuche erfolgten in einem Doppelwandgefäß mit einem zugehörigen Thermostaten, sodass eine homogene und konstante Temperatur im gesamten Reaktionsraum über die vollständige Laugungsdauer garantiert werden konnte. Durch die Zugabe einer Spatelspitze an handelsüblichem Tensid, ließ sich die Oberflächenspannung der Laugungslösung derartig adaptieren, dass sich etwaige homophobe Anteile des Feststoffs ebenfalls in die Flüssigkeit einbringen ließen. Nach der Suspendierung des Feststoffs in deionisiertem Wasser und der Temperierung der entstandenen Suspension schloss eine Stickstoffoberflächenspülung an, um ein definiertes Oxidationspotenzial im Laugungsgefäß zu garantieren und jenes durch das Vorhandensein von Restsauerstoff nicht zu verändern. Die Zudosierung an Chemikalien (z. B. Schwefelsäure) erfolgte durch temperierte Tropftrichter, um einerseits die Reaktionsgeschwindigkeit unter Kontrolle zu halten, aber auch die Temperatur im Reaktionsraum möglichst konstant zu belassen. Um die Konzentrationsunterschiede im Lösungsvolumen gering zu halten, fand die Rührung mittels Magnetstäbchen bei einer Umdrehungszahl von $350 \mathrm{rpm}$ statt. Der 


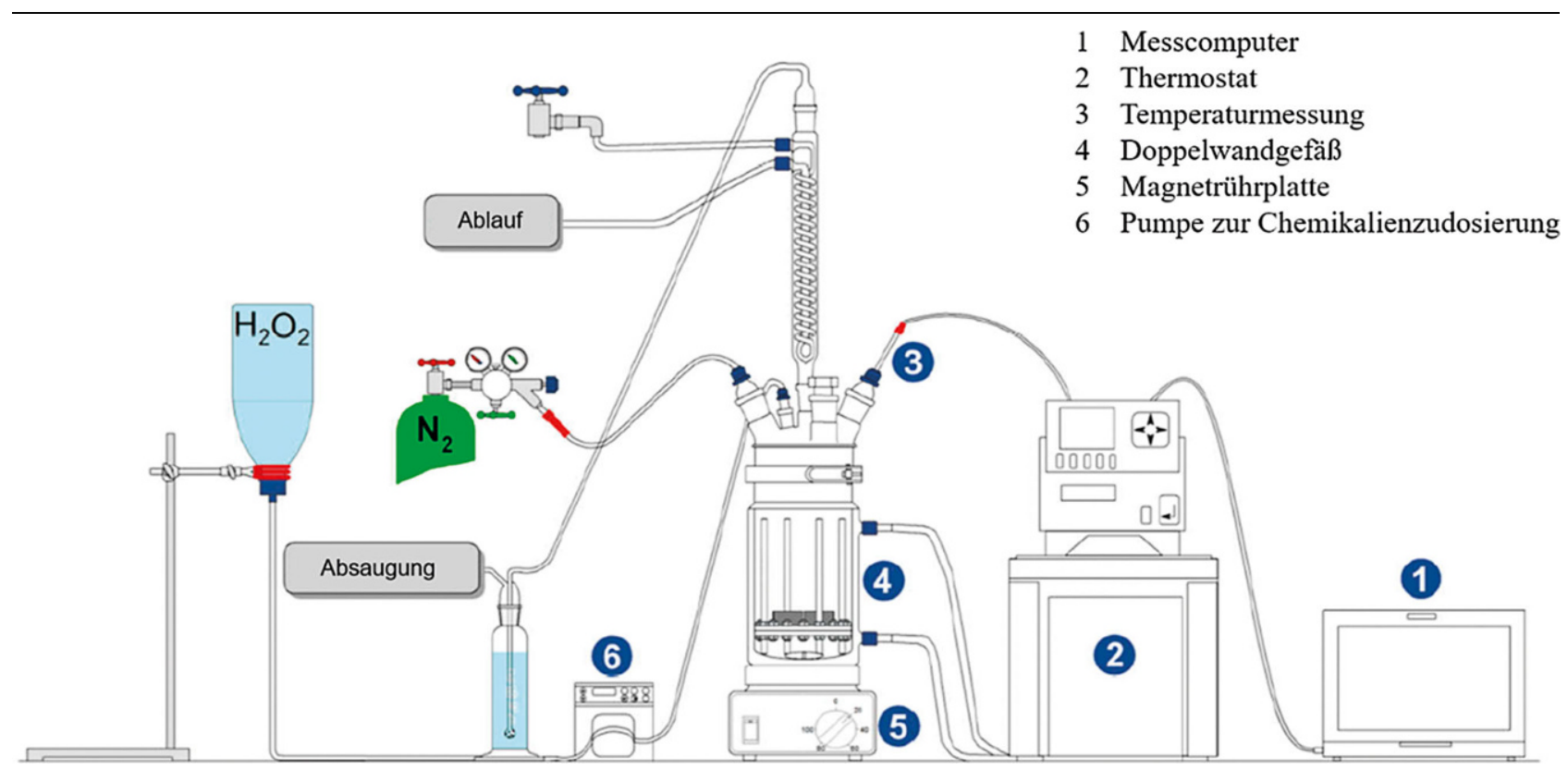

Abb. 5 Schematische Darstellung des Versuchsaufbaus für die Laugung von Müllverbrennungsaschen nach (Gerold 2021)

gesamte Versuchsaufbau ist in Abb. 5 schematisch dargestellt.

Nach der dem entsprechenden Versuch zugeordneten Haltedauer erfolgte eine Feststoff-Flüssigkeits-Trennung mittels Unterdruckfiltration. Hierzu kamen Papierfilter mit einer Maschenweite von $2-3 \mu \mathrm{m}$ zur Anwendung, um eine vollständige Abtrennung des Feststoffanteils zu realisieren.

\subsection{Ergebnisse der Laugungen von Müllverbrennungsaschen nach dem SeLiReco-Konzept}

Im Rahmen der Laugung von Müllverbrennungsaschen zeigte sich, dass eine ausführliche Parameterstudie durchaus sinnvoll und notwendig für die Bestimmung eines optimalen Laugungspunktes ist, da die Ergebnisse aus den Experimenten mit Aktivmaterialien nicht direkt übernommen werden können. Dies liegt vor allem an der unterschiedlichen Matrix und der differenten Vorbehandlung (v. a. Temperaturbereich) der beiden metallhaltigen Reststoffe.

Aufgrund der Bildung von gallertartigen Rückständen nach der Filtration der Laugungslösungen mit 2-molarer Schwefelsäure, konnte hier bisher keine Bestimmung der Zusammensetzung erfolgen. Es besteht die Annahme, dass diese Ausbildung einer dickflüssigen Phase auf die noch vorhandenen, langkettigen Kohlenstoffverbindungen oder eine Chelatbildung durch das vorhandene Eisen zurückzuführen ist. Aus diesem Grund erfolgt im Rahmen dieser Veröffentlichung ausschließlich die Auswertung der niedriger konzentrierten Lösungen (bis zu 1-molarer $\mathrm{H}_{2} \mathrm{SO}_{4}$ ).

Für die Laugung ohne Oxidationsmittel zeigt sich sehr deutlich, dass aggressive Reaktionsbedingungen wie eine hohe Temperatur und mittlere Laugungszeiten von Vorteil sind. Bei Betrachtung von hohen Feststoff-Flüssigkeits-Verhältnissen gehen die Hauptbestandteile der MV-Aschen wie Kupfer, Zink, Aluminium und Eisen bereits nach einer Laugungsdauer von $60 \mathrm{~min}$ zu einem sehr hohen Anteil in Lösung, wobei dies nur bei einer Reaktionstemperatur von $80^{\circ} \mathrm{C}$ möglich ist (siehe Abb. 6)

Die beobachteten Trends lassen sich mit jenen für die Laugung von Aktivmaterial aus verbrauchten LithiumIonen-Batterien vergleichen. Auch in diesem Zusammenhang zeigt sich, dass beispielsweise Aluminium und Eisen rasch bei hohen Säurekonzentrationen in eine gelöste Form übergehen, während das Inlösungbringen von Kobalt und Mangan deutlich mehr Zeit in Anspruch nimmt. Aus diesem Verhalten lässt sich schlussfolgern, dass eine generelle gemeinsame Aufarbeitung von unterschiedlichen metallischen Reststoffen im Allgemeinen und von Aktivmaterial und Müllverbrennungsaschen im Speziellen durchaus sinnvoll und erreichbar ist. Vor allem das ähnliche chemische Laugungsverhalten der beiden Reststoffe eröffnet die Möglichkeit zur Nutzung von Synergien und Multimetallrückgewinnung im Rahmen eines kombinierten Recyclingverfahrens.

\section{Zusammenfassung}

Im Gegensatz zum Stand der Technik ermöglicht die simultane Aufarbeitung von unterschiedlichen pulverförmigen, metallhaltigen Reststoffen ein höheres Ausbringen an Wertstoffen und führt zu selektiveren anschließenden Recyclingprozessen. Zusätzlich lassen sich durch die Zusammenführung dieser Reststoffströme Verfahren auch in Regionen etablieren, in welchen bei ausschließlicher Verwendung eines Abfallstroms keine wirtschaftliche Aufarbeitung aufgrund der anfallenden Mengen möglich wäre. Durch die gezielte Auswahl an definierten industriellen Reststoffen erfolgt die Nutzung von Synergieeffekten und damit die gemeinsame Rückgewinnung von enthaltenen Wertstoffen, wie beispielsweise Kupfer, Aluminium, Nickel, Eisen, kritische Elemente usw. Neben diesen Synergieeffekten lässt sich auch eine bedeutende Einsparung an $\mathrm{CO}_{2}$ Emissionen durch das Recycling realisieren. Dazu tragen nicht nur die verminderten Transportwege, sondern auch die Einsparung der benötigten Energie im Bereich der Erzaufbereitung und -verarbeitung bei. Die gezielte Anwendung von Recyclingstrategien, beispielsweise für Aluminium, ermöglicht somit eine beträchtliche Reduktion von Emissionen und des Energiebe- 

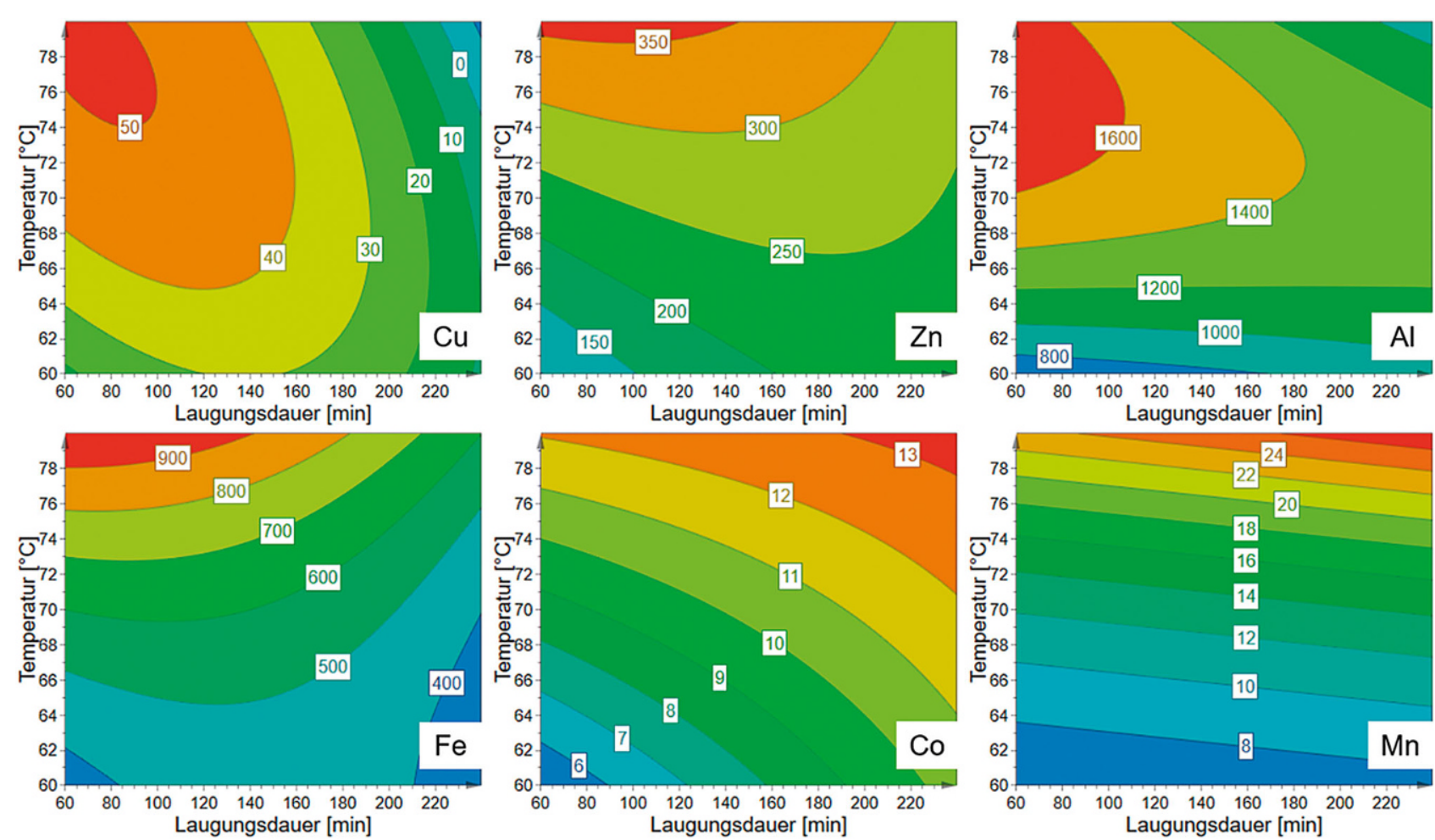

Abb. 6 Statistische Auswertung ausgewählter Laugungsversuche von Müllverbrennungsasche ohne den $Z u s a t z$ von $\mathrm{H}_{2} \mathrm{O}_{2}$ für die Metalle $\mathrm{Cu}, \mathrm{Zn}, \mathrm{Al}, \mathrm{Fe}$, Co und Mn

darfs. Des Weiteren lässt sich durch die Kombination von Aufbereitungsprozessen die Rückgewinnung von kritischen Rohstoffen aus dem Bereich der Massenmetalle realisieren und somit auch die Rohstoffverfügbarkeit deutlich verbessern.

Im Rahmen der Forschungsarbeiten am Lehrstuhl für Nichteisenmetallurgie der Montanuniversität Leoben ist es somit gelungen, die prinzipielle technische Durchführbarkeit der Laugung in Kombination von unterschiedlichen metallhaltigen Reststoffen $\mathrm{zu}$ zeigen und deren Potenzial abzuschätzen. Neben den oben beschriebenen Einsparungsmöglichkeiten im Bereich des Energiebedarfs sowohl für die Massenmetalle Kupfer und Aluminium ermöglicht die Forschung auch die Ent- bzw. Weiterentwicklung von Recyclingstrategien für Nickel und die kritischen Elemente Kobalt und Lithium. Des Weiteren führt eine Reduktion der $\mathrm{CO}_{2}$ - und Treibhausgasemissionen durch entsprechende Technologieentwicklung im Bereich der Aufbereitung von komplexen metallhaltigen Reststoffen $\mathrm{zu}$ einer nachhaltig positiven Auswirkung auf das Klima und den Rohstoffkreislauf

Funding Open access funding provided by Montanuniversität Leoben.

Open Access Dieser Artikel wird unter der Creative Commons Namensnennung 4.0 International Lizenz veröffentlicht, welche die Nutzung, Vervielfältigung, Bearbeitung, Verbreitung und Wiedergabe in jeglichem Medium und Format erlaubt, sofern Sie den/die ursprünglichen Autor(en) und die Quelle ordnungsgemäß nennen, einen Link zur
Creative Commons Lizenz beifügen und angeben, ob Änderungen vorgenommen wurden.

Die in diesem Artikel enthaltenen Bilder und sonstiges Drittmaterial unterliegen ebenfalls der genannten Creative Commons Lizenz, sofern sich aus der Abbildungslegende nichts anderes ergibt. Sofern das betreffende Material nicht unter der genannten Creative Commons Lizenz steht und die betreffende Handlung nicht nach gesetzlichen Vorschriften erlaubt ist, ist für die oben aufgeführten Weiterverwendungen des Materials die Einwilligung des jeweiligen Rechteinhabers einzuholen.

Weitere Details zur Lizenz entnehmen Sie bitte der Lizenzinformation auf http://creativecommons.org/licenses/ by/4.0/deed.de. 
Breitenstein B., Goldmann D. und B. Heitmann: NE-Metallrückgewinnung aus Abfallverbrennungsschlacken unterschiedlicher Herkunft. Mineralische Nebenprodukte und Rohstoffe (2015), 2, 255-270

Bundesministerium für Klimaschutz, Umwelt, Energie, Mobilität, Innovation und Technologie: Die Bestandsaufnahme der Abfallwirtschaft in Österreich, Statusbericht, 2020

Frischenschlager H., Karigl, B., Lampert C. Pölz W., Schindler, I., Tesar, M., Wiesenberger H. und Winter B.: Klimarelevanz ausgewählter Recycling-Prozesse in Österreich, Endberich Umweltbundesamt, 2010

Gerold E.: Entwicklung eines hydrometallurgischen Recyclingkonzeptes für Lithium-IonenBatterien. Dissertation Montanuniversität Leoben (2021)

Gerold E. et al.: Leaching of metal-containing residues from the battery sector. World of Metallurgy—Erzmetall, 5 (2019a), 267-273

Gerold E. et al.: Synergy effect at the recycling of metal containing waste from the waste industry. Proceedings of European Metallurgical Conference EMC 2019b (2019), 973-982.

Kersten M.: Emissionspotential einer Schlackenmonodeponie/Schwermetalle im Sickerwasser von Müllverbrennungsschlacken - ein langfristiges Umweltgefährdungspotential. Geowissenschaften (1996), 14, 180-185
König T.: Untersuchungen zur Sortierung von Schlacken aus Hausmüllverbrennungsanlagen. Dissertation RWTH Aachen (1993)

Korthauer R. (Hg.): Handbuch Lithium-IonenBatterien. Springer Berlin Heidelberg (2013).

Lee C. K. und K.-I. Rhee: Preparation of $\mathrm{LiCoO}_{2}$ from spent lithium-ion batteries. Journal of Power Sources, 109 (2002), 17-21.

Li J. et al.: Study of extraction and purification of $\mathrm{Ni}, \mathrm{Co}$ and $\mathrm{Mn}$ from spent battery material. Hydrometallurgy, 99 (2009), 7-12.

LI J.-h. et al.: Study of spent battery material leaching process. Transactions of Nonferrous Metals Society of China, 19 (2009), 751-755.

Li L. et al.: Recovery of metals from spent lithium-ion batteries with organic acids as leaching reagents and environmental assessment. Journal of Power Sources, 233 (2013), 180-189.

Li L. et al.: Recovery of valuable metals from spent lithium-ion batteries by ultrasonic-assisted leaching process. Journal of Power Sources, 262 (2014), 380-385.

Lück T.: Die weitergehende Aufbereitung von Müllverbrennungsschlacke nach dem Verfahren der Scherer + Kohl GmbH. Optimierung der Abfallverbrennung (2004), 1, 621-640

Reuter, B., Hendrich, A., Hengstler J, Kupferschmid S. und Schwenk M.: Rohstoffe für in novative Fahrzeugtechnologien. E-mobil BW $\mathrm{GmbH}$ - Landesagentur für neue Mobilitätslösungen und Automotive Baden-Württemberg, 2019
Schmidt M. und R. Weippert: Rückgewinnung von Metallen aus der Feinfraktion von Abfallverbrennungsaschen. Mineralische Nebenprodukte und Abfälle (2016), 3, 193-205

Velázquez-Martínez O., Valio J., Santasalo-Aarnio A., Reuter M. und Serna-Guerrero: A Critica Review of Lithium-Ion-Battery Recycling Processes from a Circular Economy Perspective. Batteries (2019), 5, 68

Weibel G., Eggenberger U., Schlumberger S. und Möder U.K.: Chemical associations and mobilization of heavy metals in fly ash from municipal solid waste incineration. Waste Management (2016), 62, 147-159

Xu J., Thomas H.R., Francis R. W., Lum K. R., Wang J. und B. Liang: A review of processes and technologies for the recycling of lithium-ion secondary batteries. Journal of Power Sources (2008), 177, 512-527

Zheng X., Zhu Z., Lin X., Zhang Y., He Y., Cao H. und Z. Sun: A Mini-Review on Metal Recycling from Spent Lithium Ion Batteries. Engineering (2018), 4, 361-370

Hinweis des Verlags Der Verlag bleibt in Hinblick auf geografische Zuordnungen und Gebietsbezeichnungen in veröffentlichten Karten und Institutsadressen neutral. 